\title{
Agomelatine is effective in reducing insomnia in abstinent alcohol-dependent patients
}

\author{
Grosshans, Martin ; Mutschler, Jochen ; Luderer, Mathias ; Mann, Karl ; Kiefer, Falk
}

\begin{abstract}
OBJECTIVES: Sleep disorders are a widespread, persistent problem among alcohol-dependent patients and have been implicated in an increased risk for alcohol relapse. The melatonin-agonist agomelatine has been shown to improve overall sleep quality without daytime sedation. METHODS: In an off-label therapeutic setting, 9 alcohol-dependent patients with chronic sleep disorders received nightly doses of between 25 and $50 \mathrm{mg}$ of agomelatine. RESULTS: After 6 weeks of agomelatine treatment, the Pittsburgh Sleep Quality Index global score for all patients had decreased significantly from a mean (SD) of 13.1 (1.7) to $7.8(1.7)(\mathrm{t}=12.8 ; \mathrm{P}=0.00)$. CONCLUSIONS: Agomelatine is a preparation that is not prone to abuse. The current pilot investigation shows that agomelatine might offer the prospect of becoming a valuable addition to the pharmacological repertoire for the treatment of alcohol-dependenceassociated insomnia.
\end{abstract}

DOI: https://doi.org/10.1097/WNF.0000000000000007

Posted at the Zurich Open Repository and Archive, University of Zurich

ZORA URL: https://doi.org/10.5167/uzh-91364

Journal Article

Published Version

Originally published at:

Grosshans, Martin; Mutschler, Jochen; Luderer, Mathias; Mann, Karl; Kiefer, Falk (2014). Agomelatine is effective in reducing insomnia in abstinent alcohol-dependent patients. Clinical Neuropharmacology, 37(1):6-8.

DOI: https://doi.org/10.1097/WNF.0000000000000007 


\title{
Agomelatine Is Effective in Reducing Insomnia in Abstinent Alcohol-Dependent Patients
}

\author{
Martin Grosshans, MD, * Jochen Mutschler, MD, † Mathias Luderer, MD, * Karl Mann, MD, * \\ and Kiefer Falk, MD*
}

\begin{abstract}
Objectives: Sleep disorders are a widespread, persistent problem among alcohol-dependent patients and have been implicated in an increased risk for alcohol relapse. The melatonin-agonist agomelatine has been shown to improve overall sleep quality without daytime sedation.

Methods: In an off-label therapeutic setting, 9 alcohol-dependent patients with chronic sleep disorders received nightly doses of between 25 and $50 \mathrm{mg}$ of agomelatine.

Results: After 6 weeks of agomelatine treatment, the Pittsburgh Sleep Quality Index global score for all patients had decreased significantly from a mean (SD) of $13.1(1.7)$ to $7.8(1.7)(t=12.8 ; P=0.00)$.

Conclusions: Agomelatine is a preparation that is not prone to abuse. The current pilot investigation shows that agomelatine might offer the prospect of becoming a valuable addition to the pharmacological repertoire for the treatment of alcohol-dependence-associated insomnia.
\end{abstract}

Key Words: alcohol dependence, insomnia, abstinence, agomelatine

(Clin Neuropharm 2014;37: 00-00)

A mong abstinent alcohol-dependent (AD) patients, sleep disorders are a widespread and persistent problem and have been associated with the risk for alcohol relapse. ${ }^{1}$ Polysomnographic characteristics of AD patients include prolonged sleep latency and decreased sleep efficiency. ${ }^{2}$ Abstinent AD patients also show abnormal evening melatonin profiles, including a peak delay of up to 90 minutes. $^{3}$ Although there is a comprehensive pharmacological repertoire for the treatment of insomnia, a history of AD significantly limits available options. Benzodiazepines are efficacious but should be considered as contraindicated because of their potential for abuse, overdose, and toxicity in combination with alcohol. ${ }^{4}$ Antidepressant medications with sedative properties such as amitriptyline and doxepin have increasingly been used to treat insomnia ${ }^{5}$ but are often lethal when taken as an overdose and/or in combination with alcohol and can induce sexual dysfunction. Mirtazapine also has sedative effects, but weight gain and/or impaired sexual function can lead to incompliance. ${ }^{6}$ In addition, several anticonvulsant drugs (such as carbamazepine and gabapentin) have sedative effects and the therapeutic potential to aid sleep. ${ }^{7}$ However, carbamazepine requires blood monitoring and is associated

*Department of Addictive Behavior and Addiction Medicine, Central Institute of Mental Health Mannheim, University of Heidelberg/Medical Faculty, Mannheim, Germany; and $†$ Department of Psychiatry, Psychotherapy and Psychosomatics, Psychiatric Hospital, University of Zurich, Zurich, Switzerland.

Conflicts of Interest and Source of Funding: The authors have no conflicts of interest to declare.

This research received no specific grant from any funding agency in the public, commercial, or not-for-profit sectors.

Authors Grosshans and Mutschler contributed equally to this work.

Address correspondence and reprint requests to Martin Grosshans, MD, Department of Addictive Behavior and Addiction Medicine, Central Institute of Mental Health, J5/68159 Mannheim, Germany;

E-mail: martin.grosshans@zi-mannheim.de

Copyright (C) 2014 by Lippincott Williams \& Wilkins

DOI: 10.1097/WNF.0000000000000007 with hepatotoxicity, whereas gabapentin has been associated with an increased risk for suicide. ${ }^{8}$ Other options include antipsychotic agents such as olanzapine that have known sedative effects, but again, associated sexual dysfunction and/or weight gain often result in incompliance. ${ }^{9}$

Agomelatine is a structural analog of melatonin and acts both as an agonist of the MT1 and MT2 receptors as well as an antagonist of $5-\mathrm{HT}_{2 \mathrm{C}}$. As such, agomelatine represents a new class of antidepressant and was approved for the treatment of major depression in 2009. ${ }^{10}$ Because of its mechanism of action on melatonin receptors, agomelatine improves overall sleep quality without daytime sedation. ${ }^{11,12}$ This effect is paralleled by changes in sleep electroencephalograms and indicates improvements in overall sleep architecture. ${ }^{12}$ Furthermore, agomelatine was significantly less likely to cause sexual dysfunction in comparison with either paroxetine $^{13}$ or venlafaxine ${ }^{14}$ and was not associated with weight gain or loss. ${ }^{15}$ Together, these factors indicate that agomelatine is potentially a valuable new addition to current treatment options for insomnia in $\mathrm{AD}$ patients.

A well-known weakness of randomized controlled premarketing trials is the exclusion of patients with addiction disorders, resulting in a frequent lack of relevant naturalistic data on newly approved substances. The purposes of this retrospective case analysis were therefore to determine whether agomelatine was well tolerated and to examine the effect of agomelatine on sleep quality in abstinent $\mathrm{AD}$ patients with chronic sleep disorders. To our knowledge, this is the first naturalistic investigation of the relationship of agomelatine to sleep quality in $\mathrm{AD}$.

\section{METHODS}

\section{Participants}

The sample consisted of 8 male and 1 female (outpatient) AD patients with persistent insomnia but without depression. All patients had no depression (mean HAMD-21 score, $<10$ ) or other psychiatric (except nicotine dependence) or neurological illnesses. The mean (SD) age of the participants was 47.2 (11.2) years, with a mean (SD) duration of AD of 20 (8.3) years. Before the current treatment, several patients were weaned off known sleep-promoting substances either because of their potential for abuse (benzodiazepines) or because they were not well tolerated, including impairment of sexual function and/or weight gain and/or daytime sedation (antidepressants, antipsychotics). Because most patients exhibited long-term AD, 7 of 9 patients received either disulfiram $(1500 \mathrm{mg} / \mathrm{wk})$ or naltrexone $(50 \mathrm{mg} / \mathrm{d})$ to prevent relapse.

Because agomelatine is potentially hepatotoxic, it was only prescribed in patients who fulfilled the following preconditions: (1) patients well known to our department with a proven history of reliability and timely and regular presentation, (2) patients who were comprehensively informed of the potential hepatotoxicity of agomelatine, particularly in combination with alcohol intake, and showed understanding of the necessity to rapidly reduce and cease agomelatine use in case of a relapse, (3) patients 
with no known liver diseases, and (4) patients who consented to repeated laboratory blood analyses before and during treatment.

\section{Agomelatine Treatment}

The study commenced with nightly oral doses of $25 \mathrm{mg}$ in all patients. After 3 weeks, all patients were revisited and, in 6 of 9 patients, the dosage of agomelatine was increased to $50 \mathrm{mg} / \mathrm{d}$ because of its only partial effectiveness on sleep quality.

\section{Assessment of Sleep Quality}

Sleep quality was assessed using the Pittsburgh Sleep Quality Index (PSQI) before (T1) and after 6 weeks of treatment (T2) with agomelatine. The PSQI is a self-rated questionnaire that assesses sleep quality and disturbances. Nineteen individual items generate 7 "component" scores: subjective sleep quality, sleep latency, sleep duration, habitual sleep efficiency, sleep disturbances, use of sleep medication, and daytime dysfunction. The sum of scores for these 7 components yields 1 global score.

\section{Laboratory Analysis}

Before (T1) and during treatment with agomelatine (T2), all patients were monitored for serum levels of the liver enzymes $\gamma$-glutamyltransferase (GGT), alanine transaminase (ALT), and aspartate transaminase (AST).

\section{Statistics}

Statistical Package for the Social Sciences 16.0 (SPSS Inc, Chicago, Ill) was used for the statistical analyses. To compare PSQI scores and laboratory values before and after 6 weeks of treatment, 1 sample $t$ test was performed. The level of significance was set at $\alpha<0.05$. Mean data values are presented, together with standard errors of the mean.

\section{RESULTS}

\section{Sleep Quality}

All patients in our sample initially scored higher than 10 (mean [SD], 13.1 [1.7]) on the PSQI global score, indicating severe insomnia. After 6 weeks of agomelatine treatment, the PSQI global score for all patients had decreased significantly, to a mean (SD) of $7.8(1.7)(t=12.8 ; P=0.00)$, (Table 1$)$.

\section{Laboratory Analyses}

Hepatotoxicity of agomelatine, as appraised by serum levels of 3 liver enzymes, was not evident at the doses used in this study (Table 1).

\section{Sexual Dysfunction and Weight Gain}

Although we did not operationalize sexual functions, no decrease of sexual desire, erectile dysfunction, and/or orgasm disorders were reported by any of the participants after 6 weeks. Furthermore, no weight gain or other drug-related adverse effects were observed.

\section{DISCUSSION}

The present retrospective data suggest that agomelatine may improve the sleep quality of male AD patients with chronic AD-associated insomnia. As expected, adverse effects such as weight gain or sexual dysfunction were not observed during 6 weeks of treatment. Thus, agomelatine was well tolerated and all patients expressed their desire to continue the treatment under the conditions described previously.

However, post-marketing experience with agomelatine has demonstrated that one adverse effect is a possible increase in serum levels of liver enzymes. This led to a recommendation by the European Medicines Agency for liver function tests as a precautionary measure at the onset of treatment, then periodically at 6 and 12 weeks as well as 12 months, and, thereafter, when clinically indicated. ${ }^{16}$ In addition, it should be noted that agomelatine is contraindicated in patients with hepatic impairment such as cirrhosis. Thus, in light of the possible liver toxicity of agomelatine in a population already vulnerable to liver disease per se, we strongly recommend that agomelatine be prescribed only to those AD patients who fulfill the criteria defined by us previously.

An important limitation in the present study is that most of the included patients have long-term alcoholism who are receiving treatment with disulfiram. Because disulfiram treatment triggers the formation of tryptophol, a compound that induces sleep, ${ }^{17}$ the presence of this compound may influence the assessment of the sleep-inducing effect of agomelatine in AD. Furthermore, it is important to mention that, in general, retrospective case series with the absence of control groups provide only weak statistical evidence and can be prone to bias. On the other hand, case series can generate new hypotheses or possible

TABLE 1. Patients Characterized by Age and Sex

\begin{tabular}{lccccccccc}
\hline Age,, , Sex & Dosage, mg & PSQI (T1) & PSQI (T2) & GGT (T1) & GGT (T2) & AST (T1) & AST (T2) & ALT (T1) & ALT (T2) \\
\hline $49(\mathrm{~m})$ & 25 & 12 & 8 & 38 & 19 & 22 & 19 & 41 & 19 \\
57 (m) & 50 & 14 & 7 & 21 & 18 & 18 & 23 & 13 & 17 \\
$58(\mathrm{~m})$ & 50 & 11 & 5 & 18 & 39 & 17 & 20 & 10 & 14 \\
45 (m) & 25 & 13 & 8 & 23 & 15 & 60 & 27 & 42 & 22 \\
$43(\mathrm{~m})$ & 50 & 11 & 7 & 30 & 27 & 23 & 27 & 28 & 24 \\
$65(\mathrm{~m})$ & 50 & 16 & 11 & 26 & 24 & 33 & 41 & 34 & 33 \\
$43(\mathrm{~m})$ & 50 & 12 & 9 & 30 & 28 & 31 & 30 & 29 & 21 \\
$33(\mathrm{~m})$ & 50 & 15 & 8 & 31 & 33 & 37 & 36 & 55 & 47 \\
$32(\mathrm{f})$ & 25 & 14 & 9 & 58 & 23 & 28 & 24 & 28 & 13 \\
Mean (SD) & - & $13.1(1.7)$ & $7.8(1.7)^{*}$ & $30.5(3.9)$ & $25.0(3.2)^{*}$ & $29.8(4.3)$ & $27.5(3.1)^{*}$ & $31.1(4.7)$ & $28.1(7.0)^{*}$ \\
\hline
\end{tabular}

Agomelatine dosage after 6 weeks.

Sleep quality and liver enzymes (U/L) before (T1) and after 6 weeks (T2).

*The statistical significance between the means of T1 and T2 $(P<0.01)$. 
new treatment options that can be later tested under controlled conditions. In conclusion, agomelatine is a preparation that is not prone to abuse, shows a robust decrease in PSQI global score in $\mathrm{AD}$ patients, and now offers the prospect of becoming a valuable addition to the pharmacological repertoire for the treatment of $\mathrm{AD}$-associated insomnia. To corroborate and extend our current data, we strongly recommend the further assessment in large controlled clinical trials.

\section{REFERENCES}

1. Brower KJ. Insomnia, alcoholism and relapse. Sleep Med Rev 2003;7 (6):523-539.

2. Brower KJ, Aldrich MS, Robinson EA, et al. Insomnia, self-medication, and relapse to alcoholism. Am J Psychiatry 2001;158 (3):399-404.

3. Kuhlwein E, Hauger RL, Irwin MR. Abnormal nocturnal melatonin secretion and disordered sleep in abstinent alcoholics. Biol Psychiatry 2003;54(12):1437-1443.

4. Ciraulo DA, Nace EP. Benzodiazepine treatment of anxiety or insomnia in substance abuse patients. Am J Addict 2000;9(4):276-279.

5. Walsh JK, Schweitzer PK. Ten-year trends in the pharmacological treatment of insomnia. Sleep 1999;22(3):371-375.

6. Anttila SA, Leinonen EV. A review of the pharmacological and clinical profile of mirtazapine. CNS Drug Rev 2001;7(3):249-264.

7. Placidi F, Scalise A, Marciani MG, et al. Effect of antiepileptic drugs on sleep. Clin Neurophysiol 2000;111(Suppl 2):S115-S119.

8. Patorno E, Bohn RL, Wahl PM, et al. Anticonvulsant medications and the risk of suicide, attempted suicide, or violent death. JAMA 2010;303 (14):1401-1409.
9. Bobes J, Rejas J, Garcia-Garcia M, et al. Weight gain in patients with schizophrenia treated with risperidone, olanzapine, quetiapine or haloperidol: results of the EIRE study. Schizophr Res 2003;62(1-2):77-88.

10. de Bodinat C, Guardiola-Lemaitre B, Mocaer E, et al. Agomelatine, the first melatonergic antidepressant: discovery, characterization and development. Nat Rev Drug Discov 2010;9(8):628-642.

11. Lemoine P, Guilleminault C, Alvarez E. Improvement in subjective sleep in major depressive disorder with a novel antidepressant, agomelatine: randomized, double-blind comparison with venlafaxine. J Clin Psychiatry 2007;68(11):1723-1732.

12. Quera Salva MA, Vanier B, Laredo J, et al. Major depressive disorder, sleep EEG and agomelatine: an open-label study. Int $J$ Neuropsychopharmacol 2007;10(5):691-696.

13. Montejo AL, Prieto N, Terleira A, et al. Better sexual acceptability of agomelatine (25 and $50 \mathrm{mg}$ ) compared with paroxetine $(20 \mathrm{mg})$ in healthy male volunteers. An 8-week, placebo-controlled study using the PRSEXDQ-SALSEX scale. J Psychopharmacol 2010;24(1):111-120.

14. Kennedy SH, Rizvi S, Fulton K, et al. A double-blind comparison of sexual functioning, antidepressant efficacy, and tolerability between agomelatine and venlafaxine XR. J Clin Psychopharmacol 2008;28 (3):329-333

15. Goodwin GM, Emsley R, Rembry S, et al. Agomelatine prevents relapse in patients with major depressive disorder without evidence of a discontinuation syndrome: a 24-week randomized, double-blind, placebo-controlled trial. J Clin Psychiatry 2009;70(8):1128-1137.

16. Valdoxan. Product Characteristics. European Medicince Agency; 2012. Available at: http://www.ema.europa.eu/docs/en_GB/document_library/ EPAR_-_Product_Information/human/000915/WC500046227.pdf. Accessed March 28, 2013.

17. Cornford EM, Bocash WD, Braun LD, et al. Rapid distribution of tryptophol (3-indole ethanol) to the brain and other tissues. J Clin Invest 1979;63(6):1241-1248. 